\title{
Artificial Intelligence: Financial Trading and Neurology of Decision
}

\section{Ana Njegovanović}

Master of Economics, Lecturer at Faculty of Biotechnology in Zagreb; Faculty of Economics and Tourism, University of J. Dobrila in Pula, Croatia.

\begin{abstract}
The present article is conceptual research as a documented importance of artificial intelligence as a transformational approach to the financial sector based on technological innovations as platforms in the development of the financial sector through the full introduction of the electronic market for algorithmic trading and highfrequency trading.

Artificial neural networks (neurons configured to perform certain tasks) are biologically inspired simulations to perform certain tasks such as clustering, classification, sample recognition. Neural networks cover all aspects of financial and investment decision making.

Research shows a strong role of artificial intelligence in making financial decisions in the real world: looking at neurological decision making as a difference in response frequencies ranging from real to hypothetical environment, pointing to the difficulties people have to imitate financial decisions in real situations when asked under hypothetical circumstances.

Artificial Intelligence is an interdisciplinary area that traces the transformational aspects of the financial global sector.
\end{abstract}

Key words: Artificial intelligence, Neural networks, Decision neurology, High-frequency trading JEL Classification: D8, D89, O31.

(C) The Authors, 2018. This article is published with open access at Sumy State University.

\section{Introduction}

How to define artificial intelligence, brilliant and powerful technology? Is it devastating? For today's neural networks, genetic algorithms and evolutionary programs are examples that show a surprising degree of selfreplication, self-management and self-organization even at the component level.

The concept of artificial intelligence was overwhelmed in the 1956s of the last century, a branch of computer science for the purpose of creating intelligent machines that work and react like humans. . The beginning of the research of artificial intelligence is related to British mathematician Alan Turing (Turing, A. "Computing machinery and intelligence." All 1950. Reprinted in Computation and Intelligence by G. Luger, edited by MIT Press, Cambridge, 1995, pp.23-46 .) who proposed a true computer intelligence indicator. Understanding the empirical evidence led to a Turing test that needed to determine whether the machine was capable of thinking. Sixty years later, artificial intelligence is characterized by numerous applications including computer games and understanding of human language, virtual personal assistants and robots that include computing viewing, listening, and sensory response responses (Pareek, Rahul, "Web Intelligence-An Emerging Vertical of Artificial Intelligence." International Journal of Engineering and Computer Science 3.12 (2012): 9430-436). Technologists have offered a wide range of prediction of the development of artificial intelligence since the beginning of application as a tool for relatively simple processes (some considered to be weak AI) to robots with similar mental human abilities - sometimes referred to as a powerful artificial intelligence (For example, Nigel, "Why We Should Not Fear AI Yet Yet." Wired May 8, 2015) and "If you think Siri is useful now, the next decade's generation of Siri will be more like JARVIS from Iron Man, with expanded capabilities to understand and answer ... In a decade, it will be normal for you to give your AI (Diamandis, Peter, "The World in 2025: 8 Predictions for the Next 10 Years." Singularity Hub, May 11, 2015. Sir Nigel Shadbolt emphasizes "what we really have, in artificial intelligence, is a spectrum of spectrum capability from programs that are smart but not as smart as us, to programs that are super smart in some areas" (The Life Scientific -Nigel Shadbolt. "Interview by J im Al-Khalili. BBC Radio 4. 14 Apr. 2015. Radio) with people, increasing human cognitive effects, or replacing people in performing routine and non-routine tasks (McCarthy, John. "What Is Artificial Intelligence?" Computer Science Department Stanford University, 28 Sept. 2001, Revised 12 Nov. 2007; Thomason, Richmond. "Logic and Artificial Intelligence." Department of Philosophy University of Michigan, 27 Aug. 2003 ). 
AI as a symbol of financial present and future affects all areas of the banking sector where the speed and accuracy of digital computing becomes widespread. Huge amount of data for analysis and measurement using digital technology and algorithms is based on modern financial business. The financial industry produces a huge amount of data through its operations, creating a need for efficient and accurate analyzes, resulting in the development of computer algorithms. In addition to countless opportunities provided by artificial intelligence, that other party can lead to potential threats in the financial sector and destabilize the market in milliseconds. Sophistication AI requires strong regulatory mechanisms for data security and market integrity. The rapid development of new technologies requires laws and policies that need to be agile in order to prevent algorithmic omissions but also the possibility of further development of innovation.

Financial markets are considered complex, evolutionary and nonlinear dynamics of the system (MacKinlay, 1988). Modern / advanced neuronal techniques for modeling nonlinearity and complex behavior within time series data (Mandelbrot, 1977) are needed. Mandelbrot and Hudson (2004, pp. 17-19) did not conduct an experiment with their research but already presented to the readers two real-life pricing charts ("what would be found in a brokerage report"), a graph depicting a computer generated fractal series, and a graph random walk series. The results of the research have shown that people are more sensitive to price change properties rather than price characteristics. It is also necessary to emphasize the existence of three reports on the discrimination of fractal structures of one-dimensional spatial graphs or contours (Gilden, Schmuckler and Clayton, 1993; Kumar, Zhou and Glaser, 1993; Westheimer, 1991). However, all three articles were limited to a small number of participants (two or eight) which does not allow generalization to large populations. Kumar, Zhou and Glaser (1993) compared the sensitivity of people to the fractal dimension of the graph to the performance of five numerical algorithms including the "grid dimension" method. Research by Kumar and Associates has revealed that people usually have lower limits of discrimination than numeric codes.

The research on financial decision-making implies the interdependence of combined methods with disciplines: decision theory / economics, neurobiology and computer science. The research is based mainly on the study of historical data (stock price history at NYSE). However, we need causes and not just correlations based on the movement of stock history. To get the cause of the experiments needed, much of the research is based on biology and brain research through images for the study of neural processes that are the basis of certain aspects of behavior such as risk taking, and the use of pharmacology with the aim of the role of neuromoduals such as dopamine (found in smart medicines like Ritalin) in decision making. To gain a complete picture, computer science is also used. Namely, the brain processes information, computer science provides formalism for brain count research. Also, research into the real world problem, algorithmic trading is based on the problems that have occurred in the real financial market. The assumed core of rational choice implies most of the economic theories, the way economists interpret human choices, is closely related to the concept of market efficiency.

Implications of Technology Artificial Intelligence and Machine Learning in the Financial Sector. Machine learning is a field of computer science that allows computers to learn without explicit programming (paraphrased by Samuel, Arthur, 1959), respectively how to build computers that automatically improve through experience. The fastest growing technical area lying at the intersection of computer science, statistics and the core of artificial intelligence and data science. The latest advancement is the development of new learning algorithms and the flow of explosions in online data availability and low cost accounting.

Artificial intelligence and machine learning are two postcards and are often used interchangeably; The broader machine concept that is capable of performing tasks and machine learning is the current application of artificial intelligence that is based on the idea that we really need to give the machines access to the data and let them learn for themselves.

The sector of the financial industry is adapting and adopting applications of artificial intelligence and machine learning. Namely, most of the applications of artificial intelligence and machine learning already exist and are fueled by technological advances, data availability, financial sector infrastructure, and demand factors such as the need for profitability, competition with other companies, and financial regulation requirements. Cases of use of artificial intelligence and machine learning are:

$>$ Financial institutions and suppliers who use the learning method and methods of learning machines to evaluate credit quality, pricing and market contracts and automate client interaction,

$>$ Institutions have optimized capital deficiencies with AI and machine learning techniques such as backward testing models and large-market trading impact analysis, 
$>$ Hedge funds, broker traders, and other companies using AI and machine learning to find signals for larger (and non-corrected) returns and optimization of trading execution,

$>$ Public and private sector institutions can use these technologies to regulate compliance, monitoring, evaluation of data quality and detection of fraud.

Many machine learning tools are based on statistical methods known to most of the researchers, including the expansion of linear regression models to solve potential millions of inputs, or by statistical techniques to compile a large data set for easy visualization. Generally, machine learning involves (automated) optimization, prediction, categorization but not causal concluding. When determining the factors that led to the yield of bond yields will not be done by machine learning but by machine learning algorithms. The implications of financial stability depend on the use of artificial intelligence and machine learning. Estimates of these implications require the considerations that tools are used to select the type of decision, at which time scales, which financial functions, and at which level integrates human involvement. Artificial intelligence and machine learning are adapted for multiple purposes:

$>$ Sensory indicators.

$>$ Signals of trading can help companies increase productivity and reduce costs, quickly scan, and make multiple-choice decisions.

$>$ AML / CFT fraud detection.

Current software applications in the world of finance used today, some of these applications use more access to artificial intelligence - not just machine learning.

Portfolio Management. The term "robo-advisor" is essentially unheard of - just five years ago, but it is now common in the financial landscape. The term is a mistake and does not include robots at all. Instead, roboconsultants (companies like Betterment, Wealthfront and others) algorithms are built to calibrate the financial portfolio's goals and tolerance at the user's risk.

Algorithmic trading. With the 1970s, algorithmic trading (sometimes referred to as "Automated Trading Systems", which is probably a more accurate description) involves the use of complex AI systems to make exceptionally fast trading decisions.

Algorithmic systems often create thousands or millions of trades in one day, so the term "high exchange trading" (HFT) is a subset of algorithmic trading. Most hedge funds and financial institutions uncover their $\mathrm{AI}$ approach to trading (for good reasons) but it is believed that machine learning and deep learning play an increasingly important role in calibrating real-time trading decisions.

Detection of Fraud. The Internet is increasingly used, and more and more valuable company data is stored online, we have a "perfect storm" for data security risk. While previous financial fraud detection systems were heavily dependent on complex and robust set of rules, contemporary fraud detection goes beyond controlling risk factors - it actively learns and calibrates new potential (or real) security threats.

\section{Loan / Insurance in Insurance -}

Insurance on insurance can be described as the perfect job for machine learning in finance, there is a great deal of concern in the industry that machines have replaced a large number of positions.

Human intuition and the way marketers analyze the market are incredibly valuable, and the importance of this intuition can not be underestimated or replaced (or at least, not at all times in the foreseeable future). They then try to use what individual people (creativity) combine their innovations with machine learning powers (finding samples and information in large data sets). Such co-operation in the 21st century creates new interfaces where traders can ask questions and theories and then enter the information they find interesting in the algorithm, which can then transform that data and restore them in a form that the trader can understand better.

New innovations lead to competitive advantage, such as increased access to proprietary data resources. Second, the less intuitive area is the process or strategy of the level of optimization of the trade and the cooperative forces of machine and human inputs. This is where many more companies spend their money and how traders estimate a model in the next period - how well does the whole process turn into a store. It is not necessary who has the best algorithm of "compelling", but how to formulate the best strategic process for building, testing, measuring and applying algorithms in an efficient manner, using machine and human power. 
So, the best finite product is a much more complicated machine than one algorithm. Collaborative machinehuman approaches are already beginning to spread in other industries that do not have the history of using machine learning (such as eCommerce) but where analysis and performance optimization and behavioral models are key to survival in a competitive environment.

Shortly: People have limited ability to make meaningful numerical data. However, AI is excellent in transforming numbers into graphs and other visual tools that can inform decision makers with or without technical knowledge. Though companies collect much more data than ever before, visualization tools provide a compact and compelling review that will help businesses improve their business in the past.

The reasons for such decisions relate to three main factors:

1. Responsibility. Many algorithms suffer from problems with black boxes, in which there is no explanation for how the algorithm made its answers. C-level leaders are undisputed blindly believing in these algorithms to make critical decisions for them, requiring greater accountability and transparency than the AI system than many can currently offer.

2. Accessibility. We care about human bias, but AI are people's products and are also sensitive to bias. Research that has recently been published in the journal Science, the word embedding, is a machine learning tool that helps computers to have a sense of language by looking at the correlation of words with other words, thus finding that words like "women" are closely related to art, while "man "Being closer to engineering professions. As AIs are" trained "in data sets that may contain bias, and computer scientists who may be biased, there is a risk that algorithms can produce overly biased results.

Prudent. Executive Directors became leaders by relying on their own estimate. Some may be less inclined to follow computer tips, even intelligent.

If we compare the human brain and AI, then the human brain can handle far more information than the fastest computer, the brain is 100,000 times more energy efficient than the computer, but the development of technology will change (estimates) around 2040 where technology will surpass human brain? Neurons are brain blocks and can shoot about 200 times per second or 200 hertz. Computer processes are measured in gigahertz: billions of cycles per second. DNA can be used to store non-biological information.

The McKinsey Global Institute estimates the automation until 2025; AI research will generate automation between $\$ 5.2$ and \$ 6.7 trillion in global economic value, advanced robotics relying on AI will generate between $\$ 1.7$ and $\$ 4.5$ trillion. Autonomous and semi autonomous vehicles will generate between 0.2 and 1.9 trillion dollars.

Financial Decision; Artificial Intelligence; Neurology of Decision; Neural Networks and Neural Modeling. The world is becoming more and more complex. Exponential technology encourages change in each sector. Understanding change and recognizing learning opportunities is a development initiative. Stanford's Studio, Artificial Intelligence and Life 2030 argues "As artificial intelligence replaces human roles, some jobs will be eliminated and new jobs will be created. This net effect on jobs is ambiguous, but the labor market will not be equally useful for everyone. Demand for some types of skills or abilities will probably be reduced, negatively affecting the level of employment and salaries of people with these skills "(https://ai100.stanford.edu/sites/default/files/).

There is growing evidence that AI has a strong role to play in decision-making in the real world (such as Facebook, Google, and Amazon), relying on AI algorithms as part of their decision-making process, Jeff Bezos, Amazon Director, "U basically, there is no institution in the world that can not be improved by machine learning "). There is a collection of surveys for the last 70 years, according to Deloitte (one of the "big four" accounting organizations and the world's largest business services network by revenue and number of experts; „Deloitte overtakes PwC as worlds biggest auccountant“, The Telegraph Retrieved, 15 Apr 2017.

Advantages of Artificial Intelligence in Business Decision Making:

Making decisions faster: In a world where the pace of business is accelerating and shows no signs of slowing down, the key to speeding up the decision-making process is the key. For example, with AI-based prices, oil companies can dynamically change the price of gas on demand, improving their margin by about $5 \%$. Similarly, merchants, travel agents and other services routinely use dynamic pricing to optimize their margins. 
Better Handling Multiple Inputs: Machines are far better than people in managing many different factors at the same time when making complex decisions, they can process much more data at once and use the probability of proposing or making the best possible decision.

Less tedious decisions: Multiple psychological studies show that individuals are forced to make more decisions over a short period of time, the quality of these decisions worsens over time. That's why supermarkets put candies and snacks into cash registers. Exhausted by all the decisions made during a shopping trip, customers are much more reluctant to resist the bunny candy at the point of sale. Algorithms do not have such weaknesses and can help executives avoid making bad decisions resulting from exhaustion.

However, in practice, there is also skepticism, many executive directors reluctant to surrender their duty to decide on predictive models and algorithms. MIT researcher Michael Scharge describes one company (not the only one) with billions of dollars, where it has been shown where using artificial intelligence can save hundreds of millions of dollars. However, the chairman of the board did not accept such a jump.

According to PwC's research, more than 2,000 business leaders, "Most executives say their next major decision will rely largely on human judgment, minds more than machines." Only $35 \%$ of the surveyed executives claim to rely mainly on internal data and to make strategic decisions.

Although it would be impossible to suggest that the chief executive officers should rely solely on AI to make the next strategic decision, business leaders lack the opportunity to make full use of AI to help make decisions.

Cognitive Scientists Richard Nisbett and Lee Ross in his book Human Inferences say "Human Judges are not just worse than optimal regression equations, they are worse than virtually any regression equation." So how business people can effectively leverage AI's power to make better decisions ? The reasons are: responsibility, bias and pride.

Artificial Neural Networks are biologically inspired simulations performed on a computer to perform certain tasks such as clustering, classification, sample recognition, and so on. So it is a network of artificial neurons configured to perform certain tasks.

The term "neural" is derived from the basic functional unit of "neurons" or nerve cells of the human (animal) nervous system that are present in the brain and other parts of the human (animal) body. Typical nerve cells of the human brain consist of four works: dendrites (receiving signals from other cells), the soma (cellular body) sums up all incoming signals to generate input data; axon (when the sum reaches the boundary value, the neuronal fire and the signal travels an axon to other neurons, the synapse (the point of interconnecting one neuron with other neurons.) The amount of signal transmission depends on the strength (synaptic weight) of the link.

Connections can be inhibitory (reduce strength) or exciting (increase strength) in nature. Generally, the neuron network is linked to billions of neurons with trillion interconnections between them.

Neural networks revolutionize almost all aspects of financial and investment decision-making. Financial companies around the world employ neural networks to address difficult tasks that involve intuitive judgment or require disclosure of data samples that avoid conventional analytical techniques. Many observers believe that neural networks will surpass even the best dealers and investors. Neural networks are already used to trade securities markets, economy forecasts, and credit risk analysis. Unlike other types of artificial intelligence, neural networks imitate to some extent the processing of human brain traits. As a result, neural networks can draw conclusions from incomplete data, recognize real-time patterns and predict the future.

Summarizing, we conclude:

1. Very nonlinear regression / classification.

2. Vectorial valuable inputs and outputs.

3. Potentially millions of parameters for evaluation.

4. Hidden layers learn intermediate views.

5. It is actively used to model distributed computing in the brain.

6. Gradient origin, local minimal problems.

7. Overcoming and how to deal with it. 
8. Many extensions.

Decision neurology. Neurology of decision making is also to be considered as a difference in response frequencies ranging from real to hypothetical environment. Namely, neuro-computer observation suggests that people are having difficulties in imitating financial decisions in real situations when asked about them under hypothetical circumstances. The analysis was performed using a computer model of the brain that consists of the relevant categories in the brain for financial decision making (basal ganglia for action selection, orbital frontal cardiac coding, and emotional headquarters of the amygdala). Dealing with the hypothetical decision points to bias toward accepting higher risk indicating that brain regions are responsive to outcome differentiation by activating the next activation in cortical-amygdal interaction in the human brain depending on whether the outcome of the decision is hypothesized or real.

Understanding how the human brain functions and which are the most important areas of knowledge and decision making are the nucleus of neurological data for human behavior. There is a need for broad knowledge of neurological processes that are active in decision-making and what other brains have the ability to accept our decisions in unwanted ways.

The brain consists of two types of brain cells, neurons and glia cells. Neurons are brain cells that can send and process data through electrical and chemical signaling via synapse; they are interconnected by trillions of paths within the brain and communicate with each other to create a large network.

The brain is divided into four limbs: frontal lobe, parietal lobe, occipital lobe, and temporal lobe. The frontal lobe is the largest of these, it is believed to control, among other things, judgment, memory, decision-making, and planning ability of the future, and most of these functions solve the so-called cerebral cortex. Namely, many advanced brain functions are found in cortical areas, unlike subcortical, since these parts of the brain are most recently developed through evolution (Tortora and Derrickson, 2011).

Basal ganglia are several cores that lie deep in the frontal lobe (Tortora and Derrickson, 2011) and part of the primitive human brain is strongly associated with various functions from which to select action or behavior to be performed from the set of possible so as to facilitate certain engine response, while pushing others; This is called action (Mink, 1996; Levine, 2009; Chakravarthy et al., 2010), carried out by the "Go" and "No-Go" signals associated with every possible action choice (Hikosaka, 1989).

Orbitofrontal cortex; The frontal lobe contains prefrontal cortex (PFC), which is divided into, among other things, dorsalateral prefrontal cortex (DLPFC), front cingulate cortex (ACC) and orbitofrontal cortex (OFC) believed to deal with consciousness, resonance and planning among others (Tortora Derrickson, 2011; Levine, 2009).

Amygdala, part of the lymphatic system, plays a key role in treating memories and emotions (Frank et al., 2009; Levine, 2007), also referred to as the emotional brain (Tortora and Derrickson, 2011).

Decision-making implications in the brain reward system include the activation of dopamine neurons in SNC (Tortora and Derrickson, 2011), where dopamine activity signals positive feedback, while lack of activity signals negative feedback (Schultz, 1992). In normal situations where we do not experience reward or punishment, dopamine is at the level of tonic (Franck and Claus, 2006). SNC designs reward information on many parts of the brain including striatum and OFC (Tortora and Derrickson, 2011). Thus, for example, the lack of dopamine activity will stimulate lateral OFC activation while simultaneously suppressing the medial OFC. In addition, dopamine reward data is projected on the striatum so that the striatum can learn to respond in a predictable rewarding manner.

Research and observation of the human brain has led to the suggestion that human brain can be considered as interconnection by neuronal signaling of information by forming a large network of neurons. Computer modeling of such networks is called neural network modeling; Neuronal network is a network of units that represent neurons. In this type of network, units are related in a similar way to neurons in the human brain, and these models are accordingly called conjunction models (Thomas and McClelland, 2008, Fodor and Pylyshyn, 1988 ).

Neural network modeling. Frank and Claus (2006) developed a model of neural network of basal ganglia (BG, the model contains the main features of the basal ganglia in the human brain, striatum, globus pallidus externa and interna- GPe and GPi respectively) with addition of orbitofocal cortex (OFC) and basolateral 
nucleus amigdale (ABL). This model has been developed using PDP +++ software, however Emergent version of this model has not yet been developed (Frank and Claus, 2006, Frank, M.J., Personal Communications, July, 2011).

In the expanded model with cortical and amygdala functions, a supplemented basal ganglia model with additional layers yields a basal ganglia model and an orbital frontal cortex (BG-OFC). Frank and Claus (2006) explicitly state that their PDP ++ is also developed directly from the BG model (Frank, 2005). Their model is exactly the model of basal ganglia with the addition of three layers; OFC med lat, OFC ctxt, and ABL, and a minor change of name to one of the layers without changing the functionality. The OFC_med lat layer in the model according to Frank and Claus (2006) is called the orbital frontal cortex, the medial and lateral portions.

The results show that the BG-OFC model behaves as predicted by the prospectus theory and has some significance for human behavior, the model demonstrates the same results as the prospect of theory as a neural network (Frank and Claus, 2006). All components are there; risk aversion and risk-demand behavior in order to increase the risk of gains and losses, the greater the emotional impact of loss rather than gain (Kahneman and Tversky, 1979). The exploratory factors behind this behavior are increasing amygdala loss activity by dictating a loss avoidance model rather than attempting to collect gains in accordance with the discovery of amygdal damage by eliminating the defiance of loss (De martino et al., 2010). Also, the model prefers a safe dopamine reward in a random manner as predicted by Kahnemen and Tversky (1979). In the amygdal activity results, clear effect on response frequencies and therefore readiness to take risks in the BG-OFC model is evident. The observed effect is an increased emotional attachment to the decision.

The size of hypothetical bias is considerably higher for tasks involving greater losses; this means that big losses are avoided by others when they are hypothetically shifted from the actual settings. Therefore, the claim of losing the aversion could be a driver of hypothetical bias. Theoretically, the aversion of loss is the reason for hypothetical bias if hypothetical cases are considered uncertain gains and losses of real values, observing that an increased uncertainty in the future is expected where the expected perception of the hypothetical reward value (Swardh, 2008). We can understand that we do not know what pleasure to expect from a hypothetical reward, while for real ones it is clearer.

Since the BG-OFC model is carefully developed to maintain human properties, the result of hypothetical bias increases the risk through a prospectus theory that can be extrapolated to real human behavior. Summarized, neurological mechanisms can interpret hypothetical and real decisions differently (Kang et al., 2011).

High-Frequency Trading. In the financial world, high-frequency trading has become a new norm. By using trading algorithms and dedicated tools, players in the stock market can execute a milestone rebate. The ultimate goal is to increase profits in an almost automated way. Nevertheless, in the past there have been some significant trades of high frequency stores, all of which have caused significant financial losses.

Technology progress over the last few years has enabled purchasing and sales to be increasingly automated, creating new strategies that were previously unfeasible. Stores can now be executed for several microseconds, one hundred thousand times faster than blinking (usually around 100 to 400 milliseconds).

As a result, high-frequency trading (HFT) - the use of sophisticated computer programs of extremely high speed for submission and cancellation of orders for small profits - has become widespread, and stock markets are one of the focus areas. The HFT market share has grown over the past decade, and the electronic market has become an integral part of securities trading.

Contrary to its early rapid expansion, the share of HFT as a fraction of the total stock market falls from the financial crisis (see figure 01). An assessment of potential factors affecting market growth may shed light on the future growth potential of the HFT industry.

Financial markets are constantly evolving, so when changes in the market system occur, it is important to study and proanalize the impact of the new development. In the 1980s of the last century, there were relevant issues of trading program (Harris et al., 1994), expansion of options market (Skinner, 1989). In the early 2000s there was algorithmic trading (Hendersohtt et al., 2008), decimals of prices (Chung et al., 2004), the introduction of electronic communication network (Huang, 2002), today is a high-frequency trading. 
The high trade frequency is a branch of algorithmic trading that focuses on generating profits using a high speed of execution. Used in areas such as trading in arbitrage, trading on the basis of signal and scalping. In larger stock exchanges, the volume of trading generated by these trades - usually proprietary traders, hedge fund managers and market makers - is significant.

What does high frequency trading mean? Technological innovations have always been a platform in the development of financial markets, through the introduction of the fully electronic market for algorithmic trading and high-frequency trading, trade has become almost automated. Defining high-frequency trading means trading on financial markets using algorithms. High-frequency trading algorithms are tools for traders who constantly monitor them by reacting to financial market changes faster than possible with the human brain. The significance of the role of high-frequency trading is still not sufficiently understandable. High-frequency trading is a platform that uses powerful high-CPU computers in a large number of transactions at high speeds (positions can be opened and immediately closed in nano seconds) Complex algorithms for multiple market analysis are used and market-based orders are executed Usually, fastest-performing traders are more profitable than those with slower execution speeds. In 2012, high-frequency trading accounted for over $50 \%$ of trading volume and over $30 \%$ of trading volume in Europe. Although these indicators speak sufficiently of the role of high-frequency trading, however, its meaning is not sufficiently understandable.

Contracts appearing in connection with high-frequency trading are manifested through several intertwined factors, ie through increased market volatility, with the dramatic fall in market value (Flash Crashes), which leads to doubts about fair trading speed. Then the security of the computer trading system; attempted theft of Goldman Sach's Algorithmic Trade Code 2009 (McGowan, 2010). It is also highlighted as a problem of the cause of increasing the volatility market. Simon Chapman (2013) in his article "Robo risky business dealers" states "even in normal market conditions, algorithms that use high-frequency trading can increase stock volatility. Authors Martinez and Roso (2011) state that price volatility is growing with fast trading competition. Boehmer, Fong and $\mathrm{Wu}$ (2012) found that higher intensity of high-frequency trading improves liquidity and efficiency, but also increases volatility. The presence of HF traders generates a period of high volatility in the market and a sharp drop in prices (Leal, Napoletano, Roventini, Fagioalo, 2014, pg.20). Also a variety of sources point to the claims that HFT has no effect on volatility in any measurability or significant method. Nicolas Bollen and Robert Whaley (2013) from Vanderbilt University in her study "Volatility of Future Markets: What Has Changed? "Provides an analysis of whether the volatility of future returns has changed over time. The study found that there is no evidence suggesting a volatility of return in e-commerce of future markets over time (Bollen, 2013, pg. 2). Jonathan Brogaard from the Northwestern University Kellog School of Management has built a hypothetical pricing route that removed HFT from the market and showed that stock volatility was roughly unchanged when HFT initiated trading eliminated and significantly more when all HFT trading was removed (J. Brogaard, 2011, pg. 2).

High frequency trading has attracted attention over the last few years and become an increasingly important element in financial markets. The term describes a set of techniques in electrical trading of stocks and derivatives where a large number of orders are injected into the market in nano seconds. Day trading traders strive to end "flat" without retaining any significant position and utilize several revenue-generating strategies, buying and selling shares at a high rate. Investigations have shown that traders hold shares for only 22 seconds on average. Traders use several different strategies including liquidity strategy, statistical arbitrage strategy, and liquidity disclosure strategy. In liquidity strategies, retailers strive at the speed of bid-ask, which is the difference between what customers are willing to pay and sellers willing to accept for trading stocks. The high volatility and speed of a large bid-ask can be oriented towards profits for merchants, while in return it ensures market liquidity and reduces the bid-ask spread for other participants by adopting the role of market-markers. Liquidity and poor dissemination are desirable because they reduce costs and improve the information effectiveness of asset prices. Traders using arbitration strategies, on the other hand, are trying to correlate price information between related stocks or derivatives and their origination prices. Detection of liquidity involves strategies that try to disclose large orders by sending small orders that could be affected by traders. The common core of all strategies is the absolute minimum latency demand only as the fastest trading high frequency company that will be able to benefit from existing capabilities.

Decision-making can be very complex and consumption sources depend on the applied algorithm. Basically, there are various default parameters and incoming variables that are compared using mathematical and statistical approaches. 
Globalization, computerization, financial market demands that market operators must handle the volume of trading. In the light of this development, growing IT investment and adaptation of more innovative technologies with the purpose of attracting orders and thus increasing profits. Because of the increase in trading volumes, the high trading frequency becomes an extremely important source of profits for spatial trading.

As a powerful tool, high trading frequency is a highly valuable tool but also dangerous. Although this tool reduces average trading costs for investors, which also poses systematic market risks, investor harm through quick run can reduce investor confidence. The discussion of high frequency trading is very emotional because it is natural that people are afraid of what they do not understand.

Due to sophistication and progress in computer power and network systems, technological innovation such as high frequency trading gained a growing economic importance to market quality but the possible systemic risks it places on financial markets. There is a public opinion of experts and regulators.

Instead of Conclusion. Artificial intelligence has affected all aspects of modern life. Some scientists are of the opinion that AI will overtake human intelligence in the near future. Thus, the notion of singularity (a point in the future when AI reaches and goes beyond human intelligence) refers to that point in time. There are many theories about the likelihood of singularity that the form could take and what impact it would have on human society and the economy. Advocates of the concept of singularity emphasize the acceleration of technology within the time scale between 2030 or 2040 . They also claim that the human mind is very complex and human intelligence is too tinted to mimic or replace with the machine.

Singularity could take pathways a) Creating intelligent machines (which would allow us to create super intelligent machines or enabling machines to redesign their own programs to enhance their own intelligence, what we call "Intelligence Explosion"); b) whole brain emulation (loading the mind); c) the advancement of human biological intelligence (genetic engineering); and d) the development of sophisticated brain and computer interfaces. The analysis suggests that they are the first two times more likely than others. The path of AI development is expanding.

Numerous experimental researches on decision-making at individuals and markets have been made, particularly interesting as people learn and solve complex problems, how people react to extreme events (collisions of financial markets) and why they tend to be over-responsive, as people deal with complex problems (innovations ) and how the robo-retailers' impact on financial markets can be improved.

Experimental studies carry out controlled laboratory experiments for the research of particular phenomena. Although this method is common in most other areas of science, it is new to finance, where research is based mainly on studying historical data (historical stock prices at NYSE). The problem with historical analysis shows that we can find out correlations in the data we get, but we do not know how to get the cause. An experiment is needed to get the cause.

The papers are based on biology. We are biological organisms and therefore we believe that many explanations on the types of phenomena we are interested in will be found in biology, especially in the brain. Using techniques such as brain imaging for the study of neural processes that are based on certain aspects of behavior, such as taking risks, are important parameters for making financial decisions. The use of pharmacology also involves the role of certain neuromodulators such as dopamine (found in many "smart medicines" such as Ritalin) in our decision-making.

The brain can be considered a computer device that processes information, and computer science provides us with a formalism to study brain counts.

Many researches lead to problems, inspired by problems in the real world, which are being studied at the laboratory, with the aim of developing solutions that we can then return to the real world. For example, the very little work we do in algorithmic trading is currently inspired by the problems that have occurred in the real financial markets.

So one of the recent discoveries is about our ability to make complex decisions. In traditional economic theory, it is assumed that when choosing people to choose the best option available to them, regardless of whether to choose between three options, or one billion options. This assumption is the core of what is called "rational choice", which means most economic theories, the way economists interpret human choices, and is closely related to the concept of market efficiency. People have long suspected that this assumption may be unrealistic. We know that if we increase the number of options making, there is less likely that people will ever make 
a choice, a phenomenon known as "load selection". But by computing theory to quantify the resources the computer needs to make the decision that people make in everyday life, it turns out that the ability of people to find the best option available is rapidly diminishing as computer resources are needed to make decisions (number of calculations, memory ), as identified by computer science. This means that people who are faced with decision-making will not be able to choose the best option because they do not have the resources they need.

\section{References}

1. Berger, Rob. (2015). 7 Robo Advisors That Make Investing Effortless. Forbes Magazine. Aggour, Kareem S., Piero P. Bonissone, William E. Cheetham, and Richard P. Messmer.

2. Brogaard, J. (2010) High frequency trading and its impact on market quality. Northwestern University Kellogg School of Management. Working Paper.

3. Diamandis, Peter. (2015). The World in 2025: 8 Predictions for the Next 10 Years. Singularity Hub.

4. De Martino, B., Camerer, C. F. and Adolphs, R. (2010). Amygdala damage eliminates monetary loss aversion. Proceedings of the National Academy of Sciences, 107, 3788-3792.

5. Frank, M. J. and Claus, E. D. (2006). Anatomy of a Decision: Striato-Orbitofrontal Interactions in Reinforcement Learning, Decision Making, and Reversal. Psychological Review, 113(2), 300- 326.

6. Frank, M. J., Cohen, M. X. and Sanfey, A. G. (2009). Multiple Systems in Decision Making: A Neurocomputational Perspective. Current Directions in Psychological Science, 18(2), 73-77.

7. Gilden, Schmuckler and Clayto, Kumar, Zhou and Glaser, Westheimer. (1993. The perception of natural contour, Psychological Review.

8. Hikosaka, O. (1989). Neural mechanisms of procedural learning.

9. Hikosaka, O. (1989). Role of basal ganglia in initiation of voluntary movements. In Arbib, M. A. and Amari, S. (Eds.), Dynamic Interactions in Neural Networks: Models and Data. New York, NY, USA: Springer-Verla, 153-167).

10. Kang, M. J., Rangel, A., Camus, M. and Camerer, C. F. (2011). Hypothetical and Real Choice Differentially Activate Common Valuation Areas. The Journal of Neuroscience, 31(2), 461-468.

11. Kulkarni, Nitish. (2016). Computer Vision Startup Third Eye Pivots From Google Glass To Mobile. TechCrunch.

12. Levine, D. S. (2007). How Does the Brain Create, Change and Selectively Override its Rules of Conduct?. In Perlovsky, L. I. and Kozma, R. (Eds.), Neurodynamics of Cognition and Consciousness Berlin, Germany: Springer-Verla, 163-181.

13. Markoff, John. (2015). A Learning Advance in Artificial Intelligence Rivals Human Abilities. The New York Time.

14. Miller, Stephen. (2011). Computer Scientist Coined 'Artificial Intelligence'. WS.

15. Miller, Greg. (2014). The Huge, Unseen Operation Behind the Accuracy of Google Maps. Wired.com.

16. McCarthy, John. (2007). What Is Artificial Intelligence? Computer Science Department Stanford University.

17. Thomason, Richmond. (2003). Logic and Artificial Intelligence. Department of Philosophy University of Michigan.

18. Nash, Kim S. (2015). PayPal Fights Fraud with Machine Learning, WSJ.

19. Naz, Mufassra, Alpha Tom Kodamullil, and Martin Hofmann-Apitius. (2015). Reasoning over Genetic Variance Information in Cause and-effect Models of Neurodegenerative Diseases. Briefings in Bioinformatics Advance Acces.

20. Nadkarni, Prakash M., Lucila Ohno-Machado, and Wendy W. Chapman. (2011). Natural Language Processing: An Introduction. Journal of the American Medical Informatics Association, 544-51.

21. B. H. Juang, L. R. Rabiner. (2005). Automatic speech recognition - A brief history of the technology development. Elsevier Encyclopedia of Language and Linguistics.

22. Pareek, Rahul. (2012). Web Intelligence-An Emerging Vertical of Artificial Intelligence. International Journal of Engineering And Computer Science.

23. Rettinger, Jonathan. (2015). How Close Are We to a Real Self-Driving Car? The Huffington Pos.

24. Mui, Chunka. (2013). Google's Trillion-Dollar Driverless Car - Part 2: The Ripple Effects. Forbes Magazine.

25. Realistic Facial Reconstructions Enhanced by Combining Three Computer Vision Methods. (2015).

26. Rettinger, Jonathan. (2015). How Close Are We to a Real Self-Driving Car? The Huffington Post, 21 Oct.; Mui, Chunka. Google's Trillion-Dollar Driverless Car -- Part 2: The Ripple Effects. Forbes Magazine. 
27. Swärdh, J-E. (2008). Hypothetical bias and certainty calibration in a value of time experiment. Stockholm: Swedish National Road \& Transport Research Institute (VTI).

28. Tortora, G. J. and Derrickson, B. (2011). Principles of Anatomy and Physiology (13th ed.). New Jersey, NJ, USA: Wiley \& Sons.

29. Thomas, M. S. C. and McClelland, J. L. (2008). Connectionist models of cognition. In Sun, R. (Ed.), Cambridge handbook of computational psychology(pp). Cambridge, MA, USA: Cambridge University Press, 23-58.

30. Yeomans, Mike. (2015). What Every Manager Should Know About Machine Learning. Harvard Business Review.

31. Zhang, F. (2010). High-frequency trading, stock volatility, and price discovery. Available at SSRN 1691679 .

32. A. Lusardi, O.S. Mitchell „The economic importance of financial literacy : theory and evidence“ J. Econ Lit., 52 ( 2014 ), 5-44.

33. Budish, Eric, Peter Cramton and John Shim (2015), The High-Frequency Trading Arms Race: Frequent Batch Auctions as a Market Design Response. Quarterly Journal of Economics 130(4), Nov 2015, pp 15471621.

34. C.M. Kuhnen „, Asymetric learning from financial information „, J. Finance 70 ( 2015 ) pp 2929-2062

35. M. Shadlen, D. Shohamy. Decision making and squential sampling from memory. Neuron 90. (2016, 927-939.

36. M. Liljholm, J. O'Dotherty. ( 2012). Contributions oft he striatum to learning, motivation and performance: an associative account. Trnds Cogn Sci 16, 467- 475

37. S. Hartzmak. (2015). The worst , the best ignoring all the rest: the rank effect and trading behavior" Rev. Financ. Stud. 28, 1024-1059.

38.Rosenblatt, Frank. ( 1958). The perceptron: a probalistic model for information storage and organization int he brain „, Pcychological review 65.6: 386.

39. Hochreiter, Sepp and J. Schmidhuber. (1997).Long short-term memory, Neural computation 9.8, 17351780

40. Hopfield, John J. (1982 ). Neural networks and physical systems with emergent collective computational abilities. Proceedings of the national academy of science 79.8 2554- 2558

41. Brogaard, Jonathan A., Terrence R. Henderschott, and Ryan Riordan. (2013). High Frequency Trading and Price Discovery, Available at: http://ssrn.com/abstract=1928510, accessed 14 November 2013.

42. Hirschey, Nicholas H. (2011). Do High-Frequency Traders Anticipate Buying and Selling Pressure? Available at https://www2.bc.edu/ taillard/Seminar_spring_2012_files/Hirschey.pdf, accessed 13 August 2012.

43. Parkes, David C. and Michael P. Wellman (2015). Economic reasoning and artificial intelligence. Science 17 Jul 2015: 34, 6245, 267-272.

44.Stanford University. (2016). One Hundred Year Study on Artificial Intelligence (AI100). 2016 Report. https://ai100.stanford.edu/sites/default/files/ai100report10032016fnl

45. Varian, Hal R. (2016). Intelligent Technology. Finance \& Development, September 2016, Vol. 53, No. 3 , IMF. http://www.imf.org/external/pubs/ft/fandd/2016/09/varian.htm

46. Holdren J P \& Smith M, Preparing for the Future of Artificial Intelligence, (Executive Office of the President of the United States, National Science and Technology Council, Committee on Technology, 2016) accessed 8 May 2017. 